\title{
The Plan of Diagnostic Systems for the First Phase of Thailand Tokamak 1*)
}

\author{
Arlee TAMMAN and Nopparit SOMBOONKITTICHAI ${ }^{1)}$ \\ Center of Advanced Nuclear Technology, Thailand Institute of Nuclear Technology (Public Organization), \\ Nakhon Nayok, 26120 Thailand \\ ${ }^{1)}$ Department of Physics, Faculty of Science, Kasetsart University, Chatuchak, \\ Bangkok, 10900 Thailand
}

(Received 29 December 2019 / Accepted 21 May 2020)

\begin{abstract}
Thailand Tokamak 1 (TT-1) will be the first tokamak in Thailand and it is donated by ASIPP, China. The main purpose of TT-1 is to develop the national human resource for promoting the Thai fusion and plasma research and education program. The TT-1 is suitable for training Thai scientists and engineers in the design and operation of a tokamak and providing the opportunity of initiating fusion and plasma researches in Thailand. As planned, the device is adopted the refurbished chamber and remaining components of the HT-6 M tokamak of ASIPP together with several new components, e.g. initially the magnetic probes, the HCN Interferometer and the CCD diagnostics. The main components of the device are assembled at ASIPP before being shipped and installed at Thailand Institute of Nuclear Technology (TINT). In this paper, the diagnostics that will be fabricated and installed as planned for the initial phase are introduced based on the on-site discussion of the device design taken placed in June 2019 at ASIPP. The schematic overview of the locations for installing the diagnostics is also reported.
\end{abstract}

(C) 2020 The Japan Society of Plasma Science and Nuclear Fusion Research

Keywords: Thailand Tokamak 1 (TT-1), magnetic sensor, flux loop, saddle loop, CCD, HCN interferometer

DOI: $10.1585 / \mathrm{pfr} .15 .2402067$

\section{Introduction}

The HT-6 M device, a small circular poloidal crosssection tokamak, has been terminated for nearly two decades, however several research works produced by the device contributed to the understanding of the tokamak physics before the following construction of the EAST tokamak. The HT-6 M [1] device was used to perform the study of plasma heating [2], MHD instabilities [3], transport process [4], and the new diagnostics for plasma profiles [5] at that time.

The Thailand Tokamak 1 (TT-1) is aimed to provide the opportunities of facility access for learning fusion technology and design, human resource training in controlling plasma, experimental planning and operating a tokamak, and conducting fusion-related research, to the researchers of Thailand and local regions. The device will be jointly designed and built by the ASIPP staffs and the Thai researchers. The device is currently planned by assembling the refurbished components of the HT-6 M device, e.g. the main vacuum vessel and the toroidal and poloidal field coils with several further brand-new components and diagnostics, i.e. the magnet power supply, the data acquisition, the vacuum pump system, the plasma density feedback control by the gas puffing system and all diagnos-

author'se-mail: arleet@tint.or.th

*) This article is based on the presentation at the 28th International Toki Conference on Plasma and Fusion Research (ITC28). tics are designed and constructed by relying on the modern technologies. This is to improve the overall device performance

Figure 1 shows the initial phase of the construction plan of the TT- 1 in terms of the timeline of the plan and the lists of the device parameters, the coil specifications and the planned-to-install diagnostics. It consists of four sub-systems: the diagnostics, the vacuum and fueling, the plasma control and data acquisition, and the magnetic coil power supply. The magnetic probe, the HCN interferometer, and the CCD diagnostics are planned to be fabricated at ASIPP in parallel with other sub-systems. The diagnostics are used to examine the quality and parameters of the device, e.g. the plasma positioning, the magnetic coil currents, and the plasma current and density, during the operation testing. Furthermore, after the construction is completed, such diagnostics assist in the investigation of the TT-1 basic operation scenarios, which is helpful for the development of the diagnostics in the later phase of the construction plan, in terms of design, validation and comparison, by Thai researchers.

\section{Magnetic Sensors}

The magnetic sensors installed on the TT1 consist of four groups characterized by the measured parameters and the probe structure as follows,

1. Rogowski coils measure the poloidal field coil current 
2020 - 2025

Design and Fabricated in Thailand

1. Soft x-ray array 2. Optical Diagnostic

3. AXUV Diagnostics 4. HXR Diagnostics

5. LP Diagnostics

6. SXPHA Diagnostic

7. ECE Diagnostic
8. Thomson Scattering
2020 - 2021

Design and Fabricated in ASIPP

1. Magnetic Probe

2. HCN Interferometer

3. CCD Diagnostic

Install in $\mathbf{2 0 2 3}$

Move to Thailand in 2022
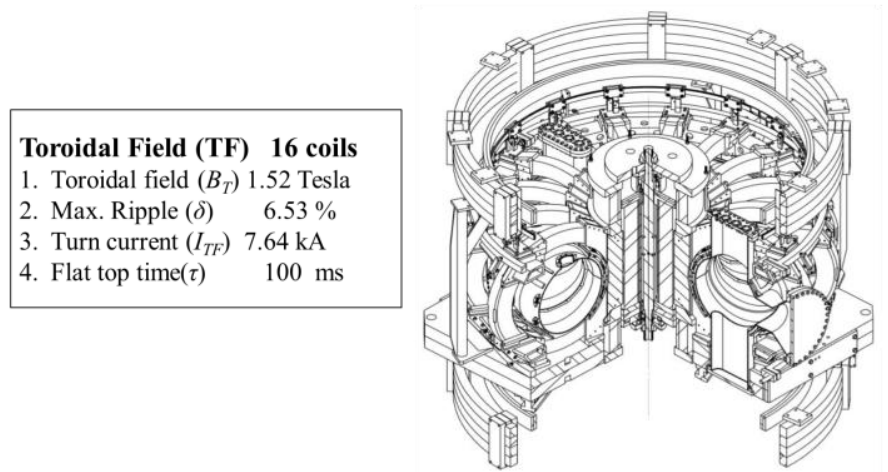

Poloidal Field (PF) 7 coils

1. Central Solenoid coils (CS 1 pieces

2. Ohmic Heating coils (HF) 4 coils

3. Vertical Field (VF) 2 coils

\begin{tabular}{|lr|}
\hline Vacuum Chamber \\
Main Radius $(R)$ & $0.65 \mathrm{~m}$ \\
Minor Radius $(a)$ & $0.2 \mathrm{~m}$ \\
\hline
\end{tabular}

Fig. 1 Main parameters and diagnostics planned to be installed in TT-1.

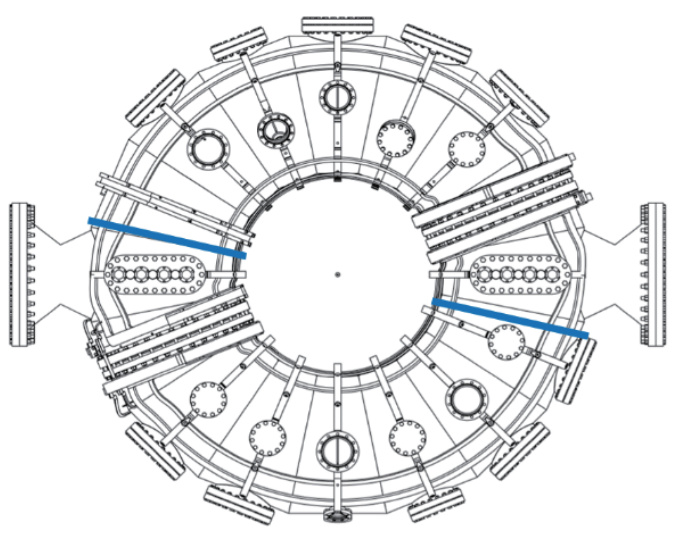

Fig. 2 Installing locations of Rogowski coils for measuring the plasma current.

and the plasma current inside the vacuum chamber. The Rogowski coils are installed at eight positions in total, i.e. six positions for measuring the magnetic field and two positions for measuring plasma current. The specifications of the Rogowski coils, which are aimed to measure the magnitude of the field, are varied depending on the types of the coils, namely the $17-\mathrm{kA}$ ohmic heating coils, the $15-\mathrm{kA}$ toroidal field coils, and the $15-\mathrm{kA}$ vertical field coils. The Rogowski coils, installed at two positions shown in Fig. 2 measure the plasma current. The maximum current allowed for the measurement is $150 \mathrm{kA}$. All Rogowski coils are designed for the minimum bandwidth of $20 \mathrm{kHz}$.

2. Mirnov coils are designed to measure the magnitude of the variable magnetic field generated by the plasma cur-

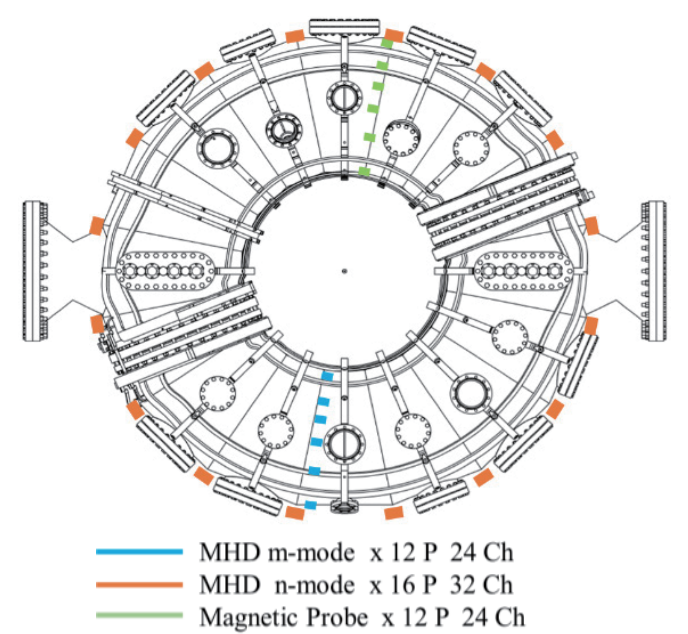

Fig. 3 Installing Positions of magnetic probes, MHD m- and nmodes for observing the MHD instability.

rent. Each one of them simultaneously allows the measurement of the magnitudes of the field which are normal to each other. Therefore, each Mirnov coil gives two output signals. The Mirnov coils act as the magnetic probes, and the probes of the $\mathrm{m}$ and $\mathrm{n}$ MHD modes. The position of the installation is shown in Fig. 3. The Mirnov coils are installed at different locations corresponding to their duties. Twelve Mirnov coils used as the magnetic probes are installed at a toroidal position but various poloidal positions. This type of Mirnov coils is designed for the minimum bandwidth of $50 \mathrm{kHz}$. The m-mode MHD probes are 


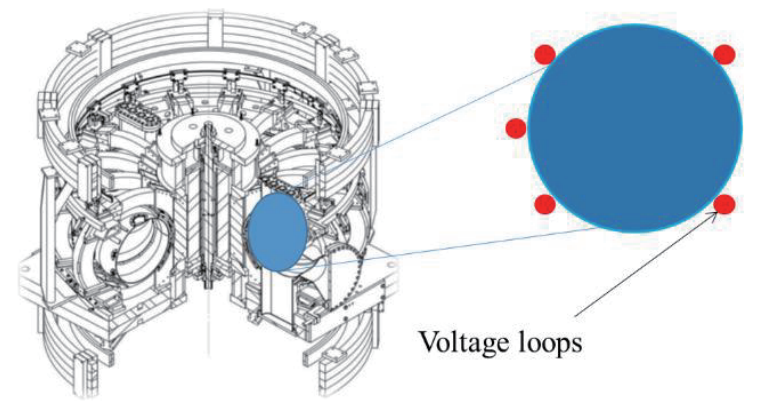

Fig. 4 Voltage loops installed at five different poloidal positions.

also located in the similar way as the magnetic probe but in a different toroidal position. Each one of sixteen Mirnov coils acting as the n-mode MHD probes is located at different toroidal positions but at the same poloidal position. The $\mathrm{m}$ and $\mathrm{n}$-mode MHD probes are designed for the minimum bandwidth of $200 \mathrm{kHz}$.

\section{Flux Loop}

In the current design, three types of flux loops are installed, i.e. diamagnetic loops, compensation loops, and voltage loops. The compensating method is applied in measuring the diamagnetic flux for monitoring the plasma energy and poloidal beta [6-9]. The diamagnetic and compensation loops are installed at a toroidal position, where one of the supported structures is specifically placed. The maximum responding frequency of the loops is $50 \mathrm{kHz}$. The voltage loops are used for monitoring the break-down loop voltage of a plasma discharge. The signals obtained from the voltage loops relate to the breakdown quality at the beginning of the plasma discharge $[10,11]$. The voltage loops are installed at five poloidal positions, each of which two loops are installed, as shown in Fig. 4. The maximum responding frequency of the loops is $50 \mathrm{kHz}$.

\section{Saddle Loop}

A saddle loop is designed to monitor the radial magnetic field, which associates with a plasma phenomenon like a magnetic island [12], in a main chamber. Therefore, the loop signal can be used to predict plasma disruption. The saddle loops of TT-1 are installed at four toroidal positions located outside the vacuum chamber shown in Fig. 5. The area covered by the loops is close to $25 \%$ of the main vacuum surface. The loop's maximum response is $50 \mathrm{kHz}$.

\section{CCD Diagnostics}

CCD high-speed camera is a powerful tool for capturing the light of the emission from the plasma inside the vacuum vessel. It is used to produce the visible image of the plasma shape and see the visible plasma phenomena, e.g. radiation by impurities and recycling near plasma facing components, MARFE at the high field side, dust mo-

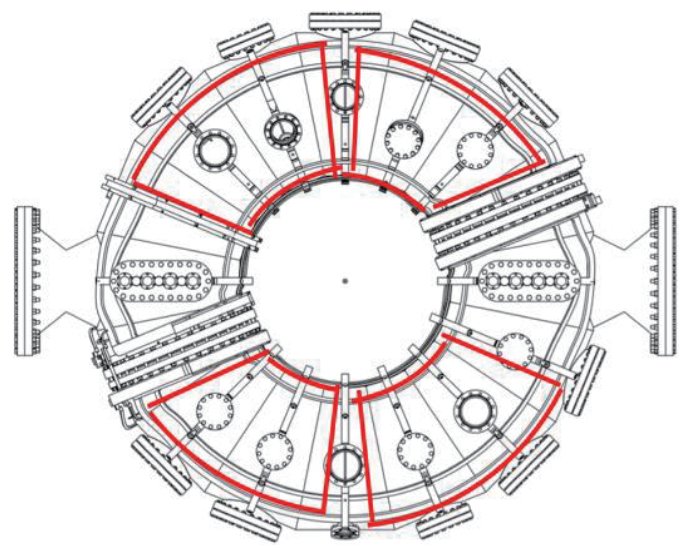

Fig. 5 Four saddle loops installed at four different toroidal positions.

tion. Figure 6 illustrates the proposed CCD diagnostics installed at three locations of the TT-1 and their viewing angles. The CCD set 1 is aimed to capture wide-angle images of plasma shape and observe overall dust motion in a long distance and MARFE at the high field side. The CCD set 2 is aimed to capture the plasma shape without the main limiter, but probably with antenna, installed in the later phase. The CCD set 3 is aimed to capture the plasma shape with the main limiter. The images obtained from the CCD set 3 not only give the information of the plasma shape but also the information of the radiation at the plasma facing component of the main limiter. Moreover, intense heat load and its associated erosion on the main limiter are probably observed. Therefore, for the initial phase, the CCD set 3 is installed. A set of images is used to analyze the plasma position and duration. Both parameters are compared with the data obtained from the magnetic probe and Rogowski coil. The rest of the CCD set, probably with an infrared CCD camera, are installed in the later phase.

\section{HCN Laser Interferometer}

This type of detector is used to measure the line integral electron density of the plasma inside the vacuum chamber along the path of the laser. The laser is aligned in three directions for measuring the average density along with three different locations. The expected density $n_{e}=$ $(1.0-2.5) \times 10^{19} \mathrm{~m}^{-3}$ in the initial phase which the operational time resolution is up to 100 microseconds. The feedback control system is used to control the plasma density inside the vacuum chamber by receiving the density value from the HCN interferometer system. The control system is con-trolled by the high-speed gas puffing technique to control the plasma density inside the vacuum chamber.

\section{Summary}

The work is to summarize the information about the on-going design of Thailand Tokamak 1 (TT-1) and its 


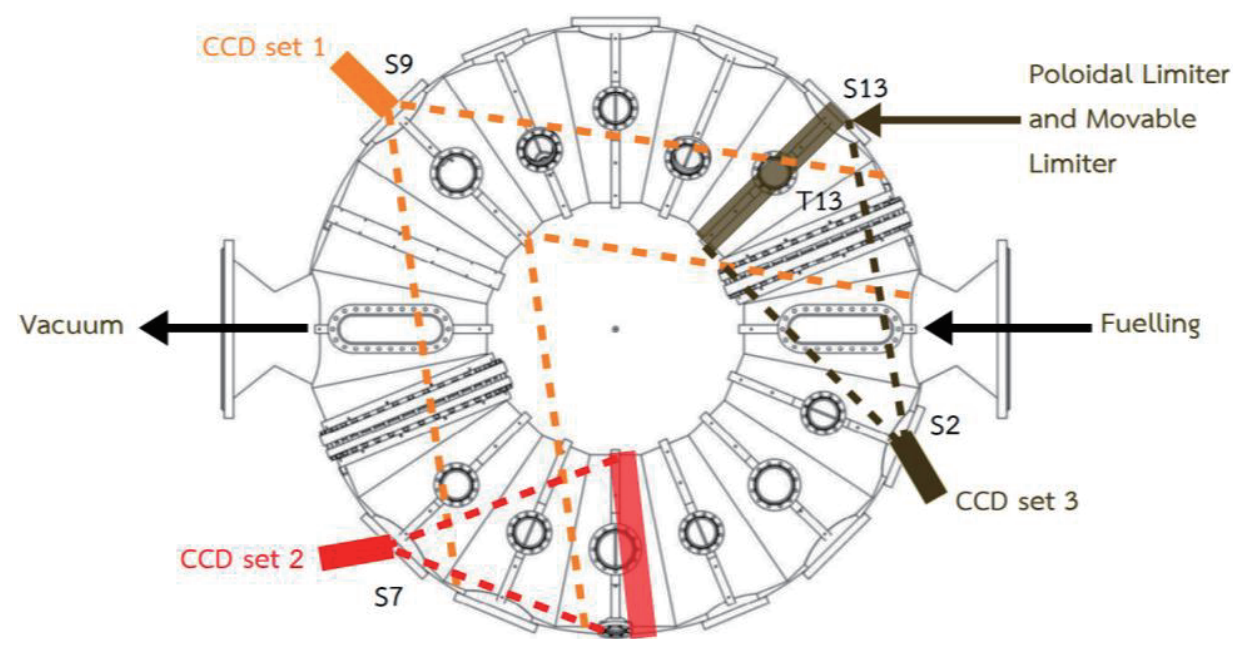

Fig. 6 The proposed CCD diagnostics installing in TT-1.

initial diagnostics during the on-site discussion at ASIPP, China in June 2019. The diagnostics need to be prepared for the initial phase of the construction plan are the magnetic probes, the $\mathrm{CCD}$ diagnostics and the HCN interferometer. The preparation of such diagnostics is carried out alongside with the construction of Thailand Tokamak 1 (TT-1), based on the HT-6 M chamber. The initial diagnostics are focused on the analysis of the TT-1 basic operation scenarios after completing the construction. In addition, they support the further development of other plasma diagnostics developed by Thai researchers.

\section{Acknowledgements}

The work was supported by Electricity Generating Authority of Thailand (EGAT) and Government Annual Research Budget through the Thailand Institute of Nuclear Technology (Public Organization). Institute of Plasma
Physics Chinese Academy of Science (ASIPP, China) donated the HT-6 M device to Thailand Institute of Nuclear Technology. Several ASIPP researchers provided the information and advice on the device design and general supervision to the Thai researchers visited ASIPP for the onsite discussion in June 2019.

[1] HT-6 M Team, Fusion Technol. 9, 476 (1986).

[2] J. Li et al., Plasma Sci. Technol. 4, 1435 (2002).

[3] J. Chen et al., Nucl. Fusion 30, 2271 (1990).

[4] W. Wang et al., Chinese Phys. Lett. 18, 793 (2001).

[5] X. Gao et al., Rev. Sci. Instrum. 66, 139 (1995).

[6] B. Shen et al., Fusion Eng. Des. 70, 311 (2004).

[7] S.K. Saha et al., Rev. Sci. Instrum. 72, 4289 (2001).

[8] D. Trembach et al., Rev. Sci. Instrum. 80, 053502 (2009).

[9] S. Kumar et al., Rev. Sci. Instrum. 81, 123505 (2010).

[10] V.K. Gusev et al., Nucl. Fusion 41, 919 (2001).

[11] P.C. De Vries et al., Nucl. Fusion 53, 053003 (2013).

[12] D. Guo et al., AIP Adv. 7, 105002 (2017). 